\title{
Prevalence and correlates of sleep disorders in Parkinson's disease: a polysomnographic study
}

\author{
Prevalência e correlatos de distúrbios do sono na doença de Parkinson: estudo \\ polissonográfico
}

Vanessa Alatriste-Booth ${ }^{1}$, Mayela Rodríguez-Violante ${ }^{2,3}$, Azyadeh Camacho-Ordoñez ${ }^{3}$, Amin Cervantes-Arriaga ${ }^{3}$

\begin{abstract}
Objective: Sleep disorders in Parkinson's disease are very common. Polysomnography (PSG) is considered the gold standard for diagnosis. The aim of the present study is to assess the prevalence of nocturnal sleep disorders diagnosed by polysomnography and to determine the associated clinical factors. Method: A total of 120 patients with Parkinson's disease were included. All patients underwent a standardized overnight, single night polysomnography. Results: Ninety-four (78.3\%) patients had an abnormal PSG. Half of the patients fulfilled criteria for sleep apnea-hypopnea syndrome (SAHS); rapid eye movement behavior disorder (RBD) was present in 37.5\%. Characteristics associated with SAHS were age $(p=0.049)$ and body mass index $(p=0.016)$. Regarding RBD, age $(p<0.001)$, left motor onset $(p=0.047)$ and levodopa equivalent dose $(p=0.002)$ were the main predictors. Conclusion: SAHS and RBD were the most frequent sleep disorders. Higher levodopa equivalent dose and body mass index appear to be risk factors for RBD and SAHS, respectively.
\end{abstract}

Keywords: Parkinson's disease, sleep disorders, polysomnography.

\section{RESUMO}

Objetivo: Os distúrbios do sono na doença de Parkinson são muito comuns. A polissonografia é considerada o padrão-ouro para o diagnóstico. 0 objetivo do presente estudo é avaliar a prevalência de distúrbios de sono noturno diagnosticados por polissonografia. Método: 120 pacientes com doença de Parkinson foram incluídos. Todos os pacientes foram submetidos a uma única noite, polissonografia de noite. Resultados: 94 (78,3\%) pacientes tiveram uma polissonografia anormal e 50\% preencheram a síndrome da apneia e hipopneia do sono (SAHOS); distúrbio de comportamento do movimento rápido dos olhos (RBD) esteve presente em 37,5\%. As características associadas com SAHOS foram idade $(p=0,049)$ e índice de massa corporal $(p=0,016)$. Quanto RBD, idade $(p<0,001)$, deixou início motor $(p=0,047)$ e levodopa dose equivalente $(p=0,002)$ foram os preditores. Conclusão: SAHOS e RBD foram os distúrbios do sono mais frequente. Dose superior equivalente de levodopa e índice de massa corporal parecem ser fatores de risco, respectivamente.

Palavras-chave: doença de Parkinson, distúrbios do sono, polissonografia.

Parkinson's disease (PD) is a neurological disorder characterized by motor symptoms such as bradykinesia, resting tremor, rigidity, impaired postural reflexes, as well as nonmotor symptoms such as hyposmia, autonomic dysfunction, neuropsychiatric symptoms, pain, and sleep disturbances ${ }^{1}$.

Sleep disorders are among the most common non-motor symptoms, with a prevalence of $60 \%$ to $90 \%^{2}$. Most frequent sleep disorders described in patients with PD are insomnia, excessive daytime sleepiness, rapid eye movement (REM) behavior disorder (RBD), sleep apnea-hypopnea syndrome (SAHS), restless legs syndrome (RLS) and periodic limb movements (PLM) ${ }^{3}$. The sleep disorders may be caused by the nocturnal motor symptoms of PD itself, adverse effects of antiparkinsonian medications or comorbid primary sleep disorders. Sleep disorders can be evaluated by anamnesis, clinical scales and neurophysiological techniques.

${ }^{1}$ Instituto Nacional de Neurología y Neurocirugía, Unidad de Medicina del Sueño, Ciudad de México, México;

${ }^{2}$ Instituto Nacional de Neurología y Neurocirugía, Clínica de Trastornos del Movimiento, Ciudad de México, México;

${ }^{3}$ Instituto Nacional de Neurología y Neurocirugía, Clínica de Enfermedades Neurodegenerativas, Ciudad de México, México.

Correspondence: Mayela Rodríguez-Violannte; Instituto Nacional de Neurología y Neurocirugía, Clínica de Trastornos del Movimiento; Insurgentes Sur \#3877 Col. La Fama; 14269 Ciudad de México, México; E-mail: mrodriguez@innn.edu.mx

Conflict of interest: There is no conflict of interest to declare.

Support: The study was funded by the National Council of Science and Technology (CONACyT) grant \#87675.

Received 02 June 2014; Received in final form 01 November 2014; Accepted 21 November 2014. 
Frequently used scales for assessment of sleep disorders include the Parkinson Disease Sleep Scale (PDSS) ${ }^{4}$, Parkinson Disease Sleep Scale -2 (PDSS-2) $)^{5}$, Scales for Outcomes in PD-Sleep (SCOPA-Sleep scale) $)^{6}$, Epworth Sleepiness Scale (ESS) ${ }^{7}$ and the Pittsburgh sleep quality index $(\mathrm{PSQI})^{8}$. Clinical evaluation may be not enough to accurately diagnose sleep disorders. Polysomnography (PSG) is considered the gold standard to diagnose and evaluate the severity of nocturnal sleep disorders ${ }^{9}$. Nocturnal psychosis and nocturia are the only items of PDSS that have been reported to correlate with some PSG parameters in patients with $\mathrm{PD}^{10}$.

The main objective of the study is to describe the prevalence of the different sleep disorders in patients with PD evaluated by PSG.

\section{METHOD}

A cross-sectional study was carried out between June 2009 and May 2013. Patients with PD according to the United Kingdom Brain Bank criteria were consecutively recruited at the Movement Disorders Clinic at the National Institute of Neurology and Neurosurgery in Mexico City.

Sociodemographic variables were collected including age, gender, education level, weight, height, drug intake, side and type of motor onset and disease severity based upon the findings of the clinical examination by the Hoehn and Yahr scale (HY) and the Unified Parkinson's Disease Rating Scale part III (UPDRS III) ${ }^{11}$. Body mass index (BMI) was calculated with the weight divided by the square of their height; overweight was defined as BMI $>25 \mathrm{~kg} / \mathrm{m}^{2}$. All patients were examined during the "on" period. Use of antiparkinsonian medication was noted and levodopa equivalent daily dose (LEDD) was calculated ${ }^{12}$. History of current use of other medications were recorded, including antipsychotics, anxiolytics, antidepressants and sleep inducers.

All patients underwent a standardized overnight, single night PSG at the Sleep Clinic using a Grass Technologies TWin (version 4.5.0.27) Polysomographer. Conventional electroencephalography electrodes (F4-M1,C4-M1,O2-M1) where placed in most patients with the exception when RBD was clinically suspected and the international 10-20 system for electrode placement was applied in order to rule out Epilepsy. Electrocardiography, chin, upper and lower extremities electromyography (EMG), electro-oculography pulse oximetry, abdominal and chest respiratory effort acquisition were also registered according to the recommended specifications of the American Academy of Sleep Medicine Manual for the Scoring of Sleep and Associated Events. PSG was later scored and analyzed by a sleep medicine specialist. The following parameters were obtained: total sleep time, sleep efficiency, percentage of time spent in non-REM $(\mathrm{N})$ and REM (R) sleep, wake after sleep onset (WASO), sleep latency, $\mathrm{R}$ sleep latency, apnea/hypopnea index, minimum and maximum oxygen saturation, minimum and maximum heart rate, cardiac dysrhythmias and periodic limb movement index. History of dream enactment behaviors, PSG recorded dream enactment behaviors and REM sleep without atonia according to the Monplasir method were also noted ${ }^{13}$. SAHS severity was defined as mild for an Apnea-Hypopnea Index $(\mathrm{AHI}) \geq 5$ and $<15$, moderate for $\mathrm{AHI} \geq 15$ and $\leq 30$, and severe for AHI $>30 / \mathrm{hr}^{14}$. PLM was classified as mild, moderate or severe according to the International Classification of Sleep Disorders ${ }^{15}$. Additionally, the diagnosis of a sleep disorder was made according to the International Classification of Sleep Disorders $2^{\text {nd }}$ edition when applicable.

\section{Patients with a non-assessable polysomnography were excluded}

The Spanish version of the SCOPA-Sleep scale was applied to all patients before performing the PSG; the sleep specialist was blinded to the scale score to avoid bias.

The study was approved by the institutional review board and ethics committee. All patients signed a written informed consent.

\section{Statistical analysis}

Measures of central tendency and dispersion for descriptive analysis were obtained. Comparison between quantitative variables was performed using a Student's t-test. Qualitative variable were compared using Chi-square test or Fisher's test as needed. Logistic regression models were performed using the presence of the sleep disorders as the dependent variables. Independent variables included in the model were gender, age, disease duration, UPDRS III score, BMI, side of motor onset and LEDD. Statistical significance was set at $\mathrm{p}<0.05$. SPSS version 17 (SPSS Inc.) was used for all analyses.

\section{RESULTS}

One hundred twenty five PD patients were initially included; five of them were excluded due to insufficient sleep time, male gender was more frequent (55.8\%). Mean age of the sample was $59.8 \pm 13$ years. Mean BMI was $27.6 \pm 4.5 \mathrm{~kg} / \mathrm{m}^{2}$, a total of $92(76.6 \%)$ patients had overweight. Mean disease duration was $6.5 \pm 6.2$ years. A total of 89 (70\%) patients had a right sided motor PD onset; motor phenotype was tremor-dominant in 76 (63.3\%) patients. The mean UPDRS III score was $28.5 \pm 17$.

Regarding PD treatment, 92 (73.6\%) were on levodopa (mean daily dose $414.6 \pm 340.5 \mathrm{mg}$ ), 88 (71.6\%) were taking a dopamine agonist, $19(15.8 \%)$ were on rasagiline and 12 
(10\%) were also receiving entacapone. Two patients were not taking any antiparkinsonian medication at the time of the study. The mean levodopa equivalent daily dose was $562.6 \pm 376.9 \mathrm{mg}$. Motor fluctuations were present in 28 (23.3\%) patients and dyskinesia was reported in 18 (15\%).

The SCOPA-Sleep scale mean score was $10.8 \pm 6.6$. According to this scale, a total of $49(40.8 \%)$ patients had diurnal sleep problems, while 47 (39.2\%) patients had sleep problems at nighttime. The most frequent sleep disorder by clinical diagnosis was insomnia in 103 (82.4\%).

\section{Overnight videopolysomnography studies}

A total of one hundred twenty PSG were included in the final analysis. Only $26(21.7 \%)$ patients had a normal PSG and $94(78.3 \%)$ an abnormal PSG. Sixty-one $(50.8 \%)$ of the patients fulfilled criteria for SAHS; mild in 31 (50.8\%), moderate in $13(21.3 \%)$ and severe in 17 (27.9\%). PLM was diagnosed in 39 patients (32.5\%); 23 (58.9\%) were classified as mild, 6 (15.4\%) as moderate and $10(25.7 \%)$ were severe. RBD was present in 48 (37.5\%) diagnosed either by history of dream enactment behavior plus $\mathrm{R}$ without atonia or capturing an episode on video-EEG. Additionally $\mathrm{R}$ sleep without atonia was noted in seven subjects (5.8\%).

Forty nine percent of the patients with a sleep disorder had a dual pathology. The most frequent association was SAHS and RBD in $15.8 \%$ of the cases. The prevalence and comorbidity of each sleep disorder are shown in Table 1.

Sleep efficiency was poor in 33 subjects $(27.5 \%)$. Other relevant polysomnographic sleep abnormalities were prolonged sleep and $\mathrm{R}$ sleep latency as well as diminished slow wave sleep. Total $\mathrm{R}$ sleep time was normal. Table 2 shows the mean values for each of the PSG parameters analyzed in the present study.

\section{Clinical correlates of sleep disorders}

Comparisons of the main demographic and clinical characteristics between PD patients with and without a PSG sleep disorder is shown in Table 3. Sleep disorders were more prevalent in males $(p=0.004)$. Regarding sociodemographic and clinical characteristics among men and

Table 1. Prevalence of sleep disorders and their comorbidities.

\begin{tabular}{lc}
\hline Sleeps disorders & $\mathrm{N}=120(\%)$ \\
\hline No sleep disorder & $26(21.7)$ \\
SAHS alone & $24(20)$ \\
RBD alone & $12(10)$ \\
PLM alone & $12(10)$ \\
SAHS + PLM & $10(8.3)$ \\
SAHS + RBD & $19(15.8)$ \\
RBD + PLM & $9(7.5)$ \\
SAHS + RBD + PLM & $8(6.7)$ \\
\hline
\end{tabular}

SAHS: Sleep apnea-hypopnea syndrome; RBD: Rapid eye movement behavior disorder; PLM: Periodic limb movements.
Table 2. Sleep architecture parameters found in the polysomnographic study.

\begin{tabular}{lc}
\hline Parameter & Mean \\
\hline Total sleep time (minutes) & $341.9 \pm 115.1$ \\
Sleep efficiency (\%) & $68.1 \pm 18.9$ \\
Sleep latency (minutes) & $41.6 \pm 59.9$ \\
R latency (minutes) & $174.9 \pm 108.1$ \\
Periodic limb movement index & $29.4 \pm 78.0$ \\
Apnea-hypopnea index (AHI) & $15.6 \pm 22.2$ \\
N1 sleep (minutes) & $16.3 \pm 24.0$ \\
N2 sleep (minutes) & $81.4 \pm 63.3$ \\
N3 sleep (minutes) & $21.3 \pm 25.9$ \\
R sleep (\%) & $20.0 \pm 21.6$ \\
\hline
\end{tabular}

R: Rapid eye movement sleep; N: Non-rapid eye movement sleep.

women with an abnormal PSG, women showed a trend to be treated more frequently with a dopaminergic agonist $(\mathrm{p}=0.05)$.

Sleep apnea-hypopnea syndrome was more prevalent in males $(65 \%$ vs $35 \%, p=0.03)$ as well as in overweight patients $(p=0.01)$. RBD was also more frequent in males (65.3\% vs $34.6 \%, \mathrm{p}=0.048)$.

In the logistic regression analysis the characteristics associated with SAHS were age $(B=1.033, p=0.049)$ and $B M I$ $(\mathrm{B}=1.120, \mathrm{p}=0.016)$. Regarding RBD, current age $(\mathrm{B}=1.071$, $\mathrm{p}<0.001)$, motor onset on the left side $(\mathrm{B}=0.400$, $\mathrm{p}=0.047)$ and LEDD $(\mathrm{B}=0.016, \mathrm{p}=0.002)$ were the main predictors. No associated factors were identified for PLM.

No statistically significant association were found between the PSG diagnosis and the SCOPA-Sleep diurnal ( $p=0.546$ ), SCOPA-Sleep nocturnal $(\mathrm{p}=0.101)$ or SCOPA-Sleep total $(\mathrm{p}=0.407)$.

\section{DISCUSSION}

Sleep disorders are common in PD and they have an impact on the patient's quality of life. Sleep disorders can present as a premotor symptom, as part of the progression of the disease or as a side-effect of the medication used in symptomatic therapy. Moreover, coexisting anxiety and depression can worsen sleep. Motor symptoms like tremor, nocturnal rigidity and hypokinesia can decrease during sleep but do not disappear completely, altering the quality of sleep. Tremor can persist during $\mathrm{N}$ sleep causing arousals; rigidity persists mainly in patients with motor fluctuations, and bradykinesia difficult the mobility ${ }^{16}$.

The overall prevalence of any sleep disturbances by PSG in our study was $78 \%$, which is similar to the $80 \%$ reported in international studies ${ }^{2,17}$. The most frequent sleep complaint was insomnia, which presented in $82 \%$ of our population. The prevalence of the other sleep disorders in our cohort was also comparable to international reports. RBD was identified in $37.5 \%$ of our population and was more common in 
male patients. This is consistent with previous reports, the frequency of RBD in patients with $\mathrm{PD}$ assessed by questionnaires or interviews ranges from $15 \%$ to $45 \%$ but when a PSG is performed the prevalence ranges from $46 \%$ to $58 \%^{18,19,20}$. The factors associated with the presence of RBD in our sample were the LEDD and left side of motor onset of disease. A study comparing patients with or without RBD reported that RBD was associated with older age, longer disease duration, and higher dose of levodopa ${ }^{21}$. Regarding the laterality of motor onset, a recent study reported a trend toward an increased frequency of RBD in PD patients who had a leftsided onset of symptoms ${ }^{22}$, while another study showed an increased frequency of nocturnal hallucinations and daytime dozing in PD patients with a left sided motor onset ${ }^{23}$. This findings suggests a pathophysiological role of the right side brain in the development of RBD in PD patients.

It should be noticed that gender was a significant variable in the bivariate analysis but not after the multivariate regression was performed, thus suggesting the presence of a covariate or effect modifier factor.

SAHS was diagnosed in half of the patients. Reports on the frequency of this disorder ranges from $27 \%$ to $60 \%{ }^{3,24,25}$. Predictors of SAHS in our sample were an older age and higher BMI. The role of BMI as a risk factor for SAHS in
PD has been well described ${ }^{26}$. Finally, PLM occurred in $32.5 \%$ patients which is also similar to other reports ${ }^{16}$. Risk factors reported in other studies include older age and severity of the disease $\mathrm{e}^{27,28}$; nevertheless none of these factors were associated with the frequency of PLM in our sample.

Polysomnographic sleep parameters disturbances associated with $\mathrm{PD}$ are poor sleep efficiency, a decrease in the quantity of $\mathrm{N} 3$ sleep, and $\mathrm{R}$ sleep ${ }^{29}$. In our patients, total N3 sleep was low but was not considered a clinical significant finding due to the fact that $\mathrm{N} 3$ diminishes with age.

SCOPA-Sleep scale did not show an association with PSG diagnoses or parameters in our study. Only PDSS have been reported to correlate with sleep efficiency as measured by $\mathrm{PSG}^{10}$; this finding should warrant the clinician to not rely only in clinical scales to diagnose a sleep disorder in PD patients. PSG should still be considered the gold standard for this purposes.

Our study has limitations. We did not include healthy controls, which makes it difficult to explain if the some sleep disorders result from the patient's age rather than disease or antiparkinsonian therapy. We cannot determine if the variety of the of the sleep disorders is higher in this particular population. A sleep latency test to assess excessive daytime sleepiness was not performed.

Table 3. Demographic and clinical characteristics of Parkinson's disease patients with and without a sleep disorder.

\begin{tabular}{|c|c|c|c|c|}
\hline & $\mathrm{N}(\%)$ & Normal PSG $(n=26)$ & Sleep disorder $(n=94)$ & $\mathrm{p}$ \\
\hline \multicolumn{5}{|l|}{ Gender } \\
\hline Male & $67(55.8)$ & $8(30.8)$ & $59(62.8)$ & \multirow[t]{2}{*}{0.004} \\
\hline Female & $53(44.2)$ & $18(69.2)$ & $35(37.2)$ & \\
\hline \multicolumn{5}{|l|}{ Education level } \\
\hline No formal & $9(7.5)$ & $1(3.8)$ & $8(8.5)$ & \multirow[t]{4}{*}{0.828} \\
\hline Elementary & $72(60)$ & $17(65.4)$ & $55(58.5)$ & \\
\hline Highschool & $15(12.5)$ & $4(15.4)$ & $11(11.7)$ & \\
\hline College & $24(20)$ & $4(15.4)$ & $20(21.3)$ & \\
\hline \multicolumn{5}{|l|}{ Side of onset } \\
\hline Right & $84(70)$ & $15(57.7)$ & $69(73.4)$ & \multirow[t]{2}{*}{0.149} \\
\hline Left & $36(30)$ & $11(42.3)$ & $25(26.6)$ & \\
\hline \multicolumn{5}{|l|}{ Motor phenotype } \\
\hline Tremor & $76(63.3)$ & $16(61.5)$ & $60(63.8)$ & \multirow[t]{3}{*}{0.792} \\
\hline Rigidity & $27(22.5)$ & 7 (26.9) & $20(21.3)$ & \\
\hline Gait disturbance & $17(14.2)$ & $3(11.5)$ & $14(14.9)$ & \\
\hline \multicolumn{5}{|l|}{ Motor status } \\
\hline Hoehn and Yahr stage (mean \pm SD) & $2.1 \pm 0.6$ & $2 \pm 0.7$ & $2.1 \pm 0.6$ & 0.913 \\
\hline UPDRS III (mean \pm SD) & $28.5 \pm 17$ & $31.6 \pm 22.8$ & $27.6 \pm 14.8$ & 0.425 \\
\hline \multicolumn{5}{|l|}{ Medication } \\
\hline Non medicated & $2(1.6)$ & $1(0.8)$ & $1(0.8)$ & 0.644 \\
\hline Levodopa & $88(73.3)$ & $17(65.4)$ & $71(75.5)$ & 0.304 \\
\hline Dopamine agonist & $86(71.6)$ & $20(76.9)$ & $66(70.2)$ & 0.506 \\
\hline MAO-B inhibitor & $19(15.8)$ & $4(15.3)$ & $15(16)$ & 0.629 \\
\hline COMT inhibitor & $12(10)$ & $5(19.2)$ & $7(7.4)$ & 0.077 \\
\hline Antipsychotics & $4(3.3)$ & 0 & $5(5.3)$ & 0.233 \\
\hline Antidepressants & $45(37.5)$ & $9(34.9)$ & $34(36.2)$ & 0.885 \\
\hline Anxiolytic & $25(20.8)$ & $5(19.2)$ & $19(20.2)$ & 0.913 \\
\hline Sleep inducers & $18(15)$ & $4(15.4)$ & $14(14.9)$ & 0.951 \\
\hline
\end{tabular}

UPDRS III: Unified Parkinson's disease Rating Scale part III. 
In conclusion, the most prevalent sleep disorders in patients with PD in our sample was insomnia, followed by SAHS, RBD and PLM. Similarities with other PSG international studies strongly suggest that PSG is reliable and important to correctly diagnose sleep disorders. Higher LEDD and higher BMI appear to be risk factors for RBD and SAHS, respectively. A left sided on motor onset of the disease also appear to be a risk factor for RBD and warrants further study.

\section{References}

1. Chaudhuri KR, Naidu Y. Early Parkinson's disease and non-motor issues. J Neurol. 2008;255(55 Suppl):33-8. http://dx.doi.org/10.1007/ s00415-008-5006-1

2. Suzuki K, Miyamoto M, Miyamoto T, Iwanami M, Hirata K. Sleep disturbances associated with Parkinson's disease. Parkinsons Dis. 2011;2011:219056. http://dx.doi.org/10.4061/2011/219056

3. Yong MH, Fook-Chong S, Pavanni R, Lim LL, Tan EK. Case control polysomnographic studies of sleep disorders in Parkinson's disease. PLoS One. 2011;6:e22511. http://dx.doi.org/10.1371/journal.pone.0022511

4. Chaudhuri KR, Pal S, DiMarco A, Whately-Smith C, Bridgman K, Mathew R, et al. The Parkinson's disease sleep scale: a new instrument for assessing sleep and nocturnal disability in Parkinson's disease. J Neurol Neurosurg Psychiatry. 2002;73:629-35. http://dx.doi.org/10.1136/jnnp.73.6.629

5. Trenkwalder C, Kohnen R, Högl B, Metta V, Sixel-Döring F, Frauscher $B$, et al. Parkinson's disease sleep scale-validation of the revised version PDSS-2. Mov Disord. 2001;26(4):644-52. http://dx.doi.org/ $10.1002 /$ mds. 23476

6. Martinez-Martin P, Visser M, Rodriguez-Blazquez C, Marinus J, Chaudhuri KR, Hilten JJ. SCOPA-sleep and PDSS: two scales for assessment of sleep disorder in Parkinson's disease. Mov Disord. 2008;23(12):1681-8. http://dx.doi.org/10.1002/mds.22110

7. Johns MW. A new method for measuring daytime sleepiness: the Epworth sleepiness scale. Sleep. 1991;14(6):540-5.

8. Buysse DJ, Reynolds CF, Monk TH, Berman SR, Kupfer DJ. The Pittsburgh Sleep Quality Index: a new instrument for psychiatric practice and research. Psychiatry Res. 1989;28(2):193-213. http://dx. doi.org/10.1016/0165-1781(89)90047-4

9. Högl B, Arnulf I, Comella C, Ferreira J, Iranzo A, Tilley B, et al. Scales to assess sleep impairment in Parkinson's disease: critique and recommendations. Mov Disord. 2010;25(16):2704-16. http://dx.doi. org/10.1002/mds.23190

10. Uemura $Y$, Nomura T, Inoue $Y$, Yamawaki M, Yasui K, Nakashima K. Validation of the Parkinson's disease sleep scale in Japanese patients: a comparison study using the Pittsburgh Sleep Quality Index, the Epworth Sleepiness Scale and Polysomnography. J Neurol Sci. 2009;287(1-2):36-40. http://dx.doi.org/10.1016/j.jns.2009.09.015

11. Fahn S, Elton RM. The Unified Parkinson's Disease Rating Scale. Recent Dev Park Dis. 1987:153-63.

12. Tomlinson CL, Stowe R, Patel S, Rick C, Gray R, Clarke CE. Systematic review of levodopa dose equivalency reporting in Parkinson's disease. Mov Disord. 2010;25(15):2649-53. http://dx.doi.org/10.1002/mds.23429

13. Lapierre O, Montplaisir J. Polysomnographic features of REM sleep behavior disorder: development of a scoring method. Neurology. 1992;42(7):1371-4. http://dx.doi.org/10.1212/WNL.42.7.1371

14. Epstein LJ, Kristo D, Strollo PJ Jr, Friedman N, Malhotra A, Patil SP, et al. Clinical guidelines for the evaluation, management and longtermr care of obstructive sleep apnea in adults. J Clin Sleep Med. 2009;5(3):263-76

15. American Academy of Sleep Medicine. International classification of sleep disorders: diagnostic and coding manual. 2nd ed. Westchester: American Academy of Sleep Medicine; 2005.

16. Grandas F, Iranzo A. Nocturnal problems occurring in Parkinson's disease. Neurology. 2004;63(8 Suppl 3):S8-11. http://dx.doi.org/ 10.1212/WNL.63.8_suppl_3.S8
17. Norlinah MI, Afidah KN, Noradina AT, Shamsul AS, Hamidon BB, Sahathevan R, et al. Sleep disturbances in Malaysian patients with Parkinson's disease using polysomnography and PDSS. Parkinsonism Relat Disord. 2009;15(9):670-4. http://dx.doi.org/ 10.1016/j.parkreldis.2009.02.012

18. Wetter TC, Trenkwalder C, Gershanik O, Högl B. Polysomnographic measures in Parkinson's disease: a comparison between patients with and without REM sleep disturbances. Wien Klin Wochenschr. 2001;113(7-8):249-253

19. Gagnon JF, Bédard MA, Fantini ML, Petit D, Panisset M, Rompré S, et al. REM sleep behavior disorder and REM sleep without atonia in Parkinson's disease. Neurology. 2002;59(4):585-9. http://dx.doi.org/ 10.1212/WNL.59.4.585

20. Iranzo A, Santamaría J, Rye DB, Valldeoriola F, Martí MJ, Muñoz E, et al. Characteristics of idiopathic REM sleep behavior disorder and that associated with MSA and PD. Neurology. 2005;65(2):247-52. http://dx.doi.org/10.1212/01.wnl.0000168864.97813.e0

21. Sixel-Döring F, Trautmann E, Mollenhauer B, Trenkwalder C. Associated factors for REM sleep behavior disorder in Parkinson disease. Neurology. 2011;7(11)7:1048-54. http://dx.doi.org/10.1212/ WNL.0b013e31822e560e

22. Baumann CR, Held U, Valko PO, Wienecke M, Waldvogel D. Body side and predominant motor features at the onset of Parkinson's disease are linked to motor and nonmotor progression. Mov Disord. 2014;29(2):207-13. http://dx.doi.org/10.1002/mds. 25650

23. Stavitsky K, McNamara P, Durso P, Harris E, Auerbach S, CroninGolomb A. Hallucinations, dreaming, and frequent dozing in Parkinson's disease: impact of right hemisphere neural networks. Cogn Behav Neurol. 2008;21(3):143-9. http://dx.doi.org/10.1097/ WNN.0b013e318185e698

24. Cochen De Cock V, Abouda M, Leu S, Oudiette D, Roze E, Vidailhet M, et al. Is obstructive sleep apnea a problem in Parkinson's disease? Sleep Med. 2010;11(3):247-52. http://dx.doi.org/10.1016/j.sleep. 2009.05.008

25. Maria B, Sophia S, Michalis M, Charalampos L, Andreas P, John ME, et al. Sleep breathing disorders in patients with idiopathic Parkinson's disease. Respir Med. 2003;97(10):1151-7. http://dx.doi. org/10.1016/S0954-6111(03)00188-4

26. Chotinaiwattarakul W, Daylau P, Chevin RD, Albin RL. Risk of sleepdisordered breathing in Parkinson's disease. Sleep Breath. 2011;15(3):471-8. http://dx.doi.org/10.1007/s11325-010-0362-3

27. Covassin N, Neikrug AB, Liu L, Corey-Bloom J, Loredo JS, Palmer BW, et al. Clinical correlates of periodic limb movements in sleep in Parkinson's disease. J. Neurol Sci. 2012;316(1-2):131-6. http://dx.doi. org/10.1016/j.jns.2012.01.004

28. Chaudhuri KR, Healy DG, Schapira AH. Non-motor symptoms of Parkinson's disease: diagnosis and management. Lancet Neurol. 2006;5(3):235-45. http://dx.doi.org/10.1016/\$1474-4422(06) 70373-8

29. Iranzo A, Valldeoriola F, Santamaría J, Tolosa E, Rumià J. Sleep symptoms and polysomnographic architecture in advanced Parkinson's disease after chronic bilateral subthalamic stimulation. J Neurol Neurosurg Psychiatry. 2002;72(5):661-4. http://dx.doi.org/ 10.1136/jnnp.72.5.661 